\title{
Cost effectiveness of a peer mentoring intervention to improve disease self-management practices and self-efficacy among African American women with systemic lupus: Analysis of the Peer Approaches to Lupus Self-management (PALS) pilot study
}

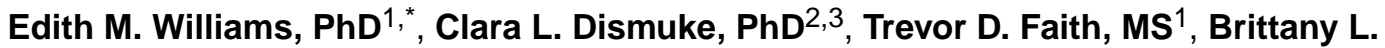 \\ Smalls, $\mathbf{P h D}^{4}$, Elizabeth Brown ${ }^{5}$, James C. Oates, MD $^{6}$, and Leonard E. Egede, MD $^{7}$ \\ ${ }^{1}$ Department of Public Health Sciences, Medical University of South Carolina, 135 Cannon Street, \\ Suite CS303, Charleston, SC 29425, USA \\ ${ }^{2}$ Veterans Health Administration, Charleston Health Equity and Rural Outreach Innovation Center, \\ Ralph H. Johnson Department of Veterans Affairs Medical Center, 109 Bee Street, Charleston, \\ SC 29401, USA
}

${ }^{3}$ College of Medicine, Center for Health Disparities Research, Medical University of South Carolina, 135 Rutledge Ave, Suite 280, Charleston, SC 29425, USA

${ }^{4}$ Center for Health Services Research, College of Medicine, University of Kentucky, $740 \mathrm{~S}$. Limestone Street, Suite J530, Lexington, KY 40536, USA

${ }^{5}$ Division of Healthcare Studies, Department of Health Professions, College of Health Professions, Medical University of South Carolina, 151-B Rutledge Ave, Charleston, SC 29425, USA

${ }^{6}$ Division of Rheumatology and Immunology, College of Medicine, Medical University of South Carolina, 96 Jonathan Lucas Street, Charleston, SC 29425, USA

${ }^{7}$ Division of General Internal Medicine, Center for Patient Care and Outcomes Research, Medical College of Wisconsin, Milwaukee, WI 53226, USA

\section{Abstract}

Objective: The Peer Approaches to Lupus Self-Management (PALS) program, was developed as a peer mentoring tool to improve health behaviors, beliefs, and outcomes in African American (AA) women with systemic lupus erythematosus (SLE). This study aims to assess the cost of the PALS intervention and determine its effectiveness when compared to existing treatments.

Methods: Peer mentors and mentees were paired on shared criteria such as life stage, marital status, or whether they were mothers. This 12 -week program consisted of a weekly peer mentoring session by telephone. Cost of healthcare utilization was evaluated by assessing the healthcare costs

\footnotetext{
*Corresponding author: Department of Public Health Sciences, Medical University of South Carolina, 135 Cannon Street, Suite 303, Charleston, SC 29425. Phone: (843)876-1519. wiled@ musc.edu.

Declaration of Conflicting Interests

The authors declare that there is no conflict of interest.
} 
pre- and post-intervention. Validated measures of quality of life, self- management, disease activity, depression, and anxiety were collected. Total direct program costs per participant were totaled and used to determine average per unit improvement in outcome measures. The benefit cost ratio and pre- versus post- intervention hospital charges were examined.

Results: 20 mentees and 7 mentors were enrolled in the PALS program. All PALS pairs completed 12 sessions lasting an average of 54 minutes. Mentees reported statistically significant decreases in patient reported disease activity, depression, and anxiety with improved trends in patient activation or patient engagement in their disease and management. The total cost per patient was $\$ 1,882.83$ and $\$ 91.14$ per patient per week. There was a savings of $\$ 23,426$ per individual receiving the intervention with a benefit cost ratio of 12.44 per patient.

Conclusion: These findings suggest that the PALS intervention was effective in improving patient-level factors and was cost-effective. Future research will need to validate these findings in a larger sample.

\section{Keywords}

Cost effectiveness; African American; women; systemic lupus erythematosus; peer mentoring

\section{INTRODUCTION}

Systemic lupus erythematosus (SLE) patients experience significantly increased healthcare costs over the course of their life due to frequent doctor visits and a complex medication regimen (1-4). The mean annual cost for SLE patients can reach up to $\$ 62,651$ with pharmaceutical costs, inpatient costs, and outpatient cost accounting for up to $30 \%, 50 \%$, and 56\%, respectively (5). This presents a major challenge for all patients diagnosed with SLE, but it is compounded in African American women who experience the disease at greater rates than any other portion of the population and are also subject to a more severe disease trajectory (6). Effective interventions to reduce disease burden, severity, and healthcare costs for African American women are needed.

African American women with SLE have been previously documented as receptive towards peer mentoring interventions in other chronic diseases, but it is a modality that has not been previously applied either in research or clinical care (7). In other low income and minority populations peer mentoring interventions have resulted in notable improvements in behaviors such as breast feeding, smoking cessation, and increased physical activity $(8,9)$ along with improved medication adherence and blood glucose monitoring in trials of people with diabetes $(10,11)$. These studies highlight the potential of peer mentoring as a culturally sensitive means to improving health behaviors and outcomes in low income and minority groups.

Moreover, previous behavioral intervention studies have not achieved targeted health outcomes in rheumatic conditions, including positive affect, sleep, social coping, and perception of bodily pain $(12,13)$. However, there is a newly developed intervention, the Peer Approaches to Lupus Self-Management (PALS) program, which was developed as a 
targeted peer mentoring approach to improving health behaviors, beliefs, and outcomes in African American women with SLE.

The current study aims to assess the cost of the PALS intervention, both direct and indirect, and determine its effectiveness when compared to existing treatments. The study team hypothesized that the PALS program would result in an overall cost savings while offsetting some standard treatment costs.

\section{MATERIALS AND METHODS}

\section{Participants}

PALS peer mentors and mentees were recruited separately. All peer mentors and mentees were of African American race/ethnicity and female gender, had a clinical diagnosis of SLE from a physician, were 18 years of age or older, and were able to communicate in English. Mentors were required to have a disease duration > 2 years; able to attend scheduled training sessions; at least some college education due to their role that involves counseling, modeling, and delivering education; and demonstration of a high level of coping/selfefficacy (score $>/=7$, range $=0-10$ ) as assessed by the Arthritis Self-Efficacy Scale (14). Competence, maturity, emotional stability, and verbal communication skills were determined by the PI prior to participating as a peer mentor, using interview questions that incorporated validated scales (15) and observation during role-playing interactions. After mentors completed their screening interview and training, they were each matched with a maximum of three mentees. Mentees were required to have a lower level of coping/self-efficacy (score $<7$, range $=0-10$ ) as assessed by the Arthritis Self-Efficacy Scale (14), but there were no criteria regarding mentees' disease duration.

\section{Intervention}

After initial enrollment and baseline assessments, peer mentors and mentees were paired based on shared criteria such as life stage, marital status, or whether they were mothers, in order to encourage compatibility. The PALS program consisted of 12 weeks of peer mentoring that included one standard educational session by telephone for approximately 60 minutes every week. The weekly educational sessions were structured in three parts: introduction, structured education, and problem solving. Weekly content was adapted from the six modules of the Chronic Disease Self-Management Program (CDSMP) (16), Arthritis Self-Management Program (ASMP) (17), and Systemic Lupus Erythematosus Self-Help (SLESH) Course (18). The material was further tailored to African American women with six added sessions based on cultural issues reported as important to African Americans in earlier research conducted by the PI (19-21). A description of the weekly content can be found in Table 1.

\section{Data Collection}

Mentees were assessed during study visits at baseline, mid-intervention (6 weeks postenrollment), and immediately following the intervention (12 weeks post-enrollment). Primary outcomes included quality of life as measured by the lupus quality of life questionnaire (LUP-QOL) (22), a lupus-specific health-related quality of life (HRQoL) 
questionnaire consisting of 34 items grouped in 8 domains: physical health, pain, planning, intimate relationships, burden to others, emotional health, body image and fatigue; selfmanagement as measured by the Patient Activation Measure (PAM) (23); and disease activity measured by two methods: 1) patient self-report by the Systemic Lupus Activity Questionnaire (SLAQ) (24) and 2) immunologic evidence as Th1/Th2 cytokine balance in blood draws that were conducted at baseline and directly following the intervention (a detailed description of this methodology has been reported elsewhere) (25). Depression was measured using the PHQ-9, a brief questionnaire that scores each of the 9 DSM-IV criteria for depression as "0" (not at all) to "3" (nearly every day) (26). Anxiety was measured using the 7-item General Anxiety Disorder (GAD) scale (GAD-7) (27). Other predictor variables assessed are described in Williams et al. (2018)(15).

\section{Cost of Implementing Intervention Program}

To estimate the cost of the intervention per individual, we totaled salary of the mentors, overhead cost for the academic medical center, and supplies/equipment required to conduct the intervention (28). In order to estimate the effectiveness (depression, anxiety, disease activity) and benefits (hospitalization charges offsets) of PALS, we examined the hospitalization charges two months pre- and post-intervention for the 20 individuals diagnosed with SLE for whom all outcome measures were reported. We first totaled the hospitalization charges for the two-month time period incurred for each individual in the study at one academic medical center prior to the intervention. We then totaled the hospitalization charges for the two-month time period incurred for each individual at the academic medical center after they completed the intervention.

\section{Statistical Analyses}

The primary outcome of economic evaluation is to estimate: $\left(\mathrm{C}_{\text {new }}-\mathrm{C}_{\mathrm{ususalcare}}\right) /\left(\mathrm{E}_{\text {new }}\right.$ $\mathrm{E}_{\text {usualcare }}$ ) where $\mathrm{C}$ is cost and $\mathrm{E}$ is effectiveness. The benefit cost ratio (BCR) was assessed as the ratio between discounted value of incremental benefits to discounted value of incremental costs. These methods are approved by the World Health Organization (WHO) and the US Guidelines for Cost-Effectiveness Analysis $(29,30)$. Student $t$ tests were conducted to examine the differences in depression, anxiety, disease activity scores, and hospitalization charges pre- and post-intervention. We used STATA version 15 and set significance at $\mathrm{p}<0.05$.

\section{RESULTS}

\section{Baseline Characteristics and Adherence to Intervention}

Table 2 shows the baseline characteristics of the 23 mentees and 10 mentors enrolled in the peer mentoring program. Three mentees and one mentor were lost to follow-up over the course of the study, and two mentors remained as back-ups, but did not actively mentor participants. Our resulting analytical sample included 20 mentees and 7 mentors. Six mentors were each matched with three mentees, and one mentor was matched with two mentees. The majority of mentees and mentors were older than 35 years, unmarried, and college-educated. About $22 \%$ of mentees had a pre-existing diagnosis of lupus nephritis or reported other indicators of renal involvement, and approximately $17 \%$ of mentees reported 
a recent history (last 3 months) of severe flares. Every mentoring pair completed all 12 of the sessions; however, 17 were delayed and took place later than originally scheduled due to scheduling conflicts. Mentors made an average of 1.24 calls to their mentees each week. Once contact was made, sessions lasted an average of 54.1 minutes (range 0-105 min). Although the option was available for additional in-person contact outside of weekly phones sessions, travel burden precluded this for all pairs.

\section{Significant Findings}

According to findings reported in Williams et al. (2018), at post-intervention, mentees reported statistically significant decreases in patient-reported disease activity (change score of 24.70 or $25 \%$ change in patient global assessment of overall lupus disease activity, $\mathrm{p}<0.001)$ and improved trends in patient activation or patient engagement in their disease and management. We also observed statistically significant decreases in depression (change score of 2.62 or $11 \%$ change in Personal Health Questionnaire-8 (PHQ-8) score, $\mathrm{p}=0.05$ ) and anxiety (change score of 3.52 or $15 \%$ change in Generalized Anxiety Disorder-8 (GAD) score, $\mathrm{p}=0.018$ ) (15). As reported in Williams et al. (2017), analyses to detect correlations between patient reported outcomes and immunologic markers associated with disease activity revealed strongest correlations between the Generalized Anxiety Disorder measure and Th1/Th2 cytokine balance and weaker correlations between depression and the Th1/ Th2 cytokine balance (25).

\section{Costs of the Intervention Program}

Table 3 shows cost per patient and cost per patient per week, accounting for staff salaries, project overhead, supplies and equipment. The largest cost categories per patient were for participant incentives and cell phones. The total cost per patient was $\$ 1,882.83$ and $\$ 91.14$ per patient per week. The effectiveness of the intervention was demonstrated by significant depression, anxiety and disease activity score decreases shown in Table 4 . The costeffectiveness ratio for anxiety was $\$ 3,423$ per 1 unit decrease in the GAD score. The costeffectiveness ratio for depression was $\$ 818$ per 1 unit decrease in the PHQ-8 score. The costeffectiveness ratio for disease activity was $\$ 85.74$ per 1 unit decrease in the SLAQ score. The benefits of the intervention consisted of the difference in pre- and post- two-month hospitalization charges for each individual who received the intervention. There was a statistically significant difference in pre-hospitalization charges ( $\$ 24,289,95 \%$ CI: $\$ 55$, $\$ 48,524)$ and post-hospitalization charges $(\$ 872,95 \%$ CI: $-\$ 324, \$ 2,070)(\mathrm{p}=0.047)$. This represents a substantial mean charge savings of $\$ 23,426$ per individual receiving the intervention. The benefit cost ratio was 12.44 per patient which implies that benefits were over 12 times higher than the cost per patient.

\section{DISCUSSION}

Our findings indicate that the PALS intervention was cost-effective, compared to standard treatment. The overall intervention cost per person was $\$ 1,882$, with each of the 12 sessions lasting on average 54 minutes. Compared to standard treatment, PALS offers $\$ 23,426$ cost savings per person following three months of intervention, according to pre- and postintervention hospitalization costs. In addition to cost savings, the intervention resulted in 
statistically significant decreases in patient-level factors such as depression, anxiety, and self- reported disease activity. Therefore, the PALS intervention appears to reduce disease burden, as well as healthcare costs.

Our results are consistent with studies, of predominantly low income and minority populations, showing that peer mentors help support healthy behaviors including breast feeding, smoking cessation, increased physical activity, and maintenance of weight loss (3135), along with improved medication adherence and blood glucose monitoring in trials of people with diabetes $(10,11,36-38)$. Until now, the extent to which peer mentors are effective in African American women with lupus had yet to be determined. PALS is the first peer mentoring intervention targeting African American women with lupus. This novel peer mentoring program built on previous literature outlining the potential benefits of peer mentoring in African American women living with lupus (39), and incorporated reciprocal relationships, mechanism for creating a complimentary social support network, sharing of knowledge and experience, and reinforcement of necessary self-management skills that led to improved outcomes (39).

These benefits are powerful because lupus disproportionately impacts African American women, and it is important to be aware of promising approaches that may minimize this disparity, while still being cost effective due to the use of peer social networks. The potential of this approach to cut costs is particularly meaningful in our study sample, since nearly a quarter of our participants reported renal involvement and recent severe flares. These characteristics are traditionally associated with higher costs. Khamashta et al. (2014) observed that patients with severe SLE had 2.2 times higher costs than patients with nonsevere disease. SLE renal involvement and severe flares were associated with higher annual direct costs, indicating the need for early intervention to minimize renal disease impact and mitigate long-term costs (40). SLE patients diagnosed earlier experience lower flare rates, less healthcare utilization, and lower costs (41), and the peer mentoring approach also has the potential to encourage earlier and more comprehensive participation in the health care system.

Peer mentoring can have positive effects on cost, utilization, and health outcomes, and it may also increase access to culturally sensitive care for African American women living with lupus. Individuals tend to have less access to care based on several factors, including lack of transportation, childcare, or health insurance and also issues surrounding cultural or historical events (42). These issues compounded with lack of access to care are sometimes magnified for minorities (43) and people living with lupus (44). According to the Agency for Healthcare Research and Quality (2017), one component of receiving access to quality care is having communication and trust with health care providers (45). Peer mentoring may promote culturally sensitive care and allow minority women to discuss aspects of care they may not feel comfortable discussing with health care providers due to distrust in health care systems and providers. Women living with lupus mentioned how a peer supporter may offer help from feelings of isolation because they felt they could not discuss certain matters with their physician (7). One focus group participant mentioned how she felt the physician was impersonal and rushed her (7). The PALS peer mentoring program may, in fact, provide a 
more convenient approach to accessing culturally sensitive care from supporters, ultimately increasing access to care, support, and health information regarding the disease.

Another benefit of the PALS peer mentoring program is the telehealth approach to providing support for African American women living with lupus. The characteristics of telehealth (e.g. remote conferencing) may increase access to care, reduce travel burdens, and enhance patient satisfaction regarding convenience and access to health information. Telehealth programs geared towards patients with chronic illnesses have seen increases in patient satisfaction for those living with asthma (46) and sleep apnea $(47,48)$. The PALS peer mentoring program has multiple advantageous features, including the telehealth component, culturally sensitive care, and patient-centered care that can impact various outcomes like access to health information, patient satisfaction, and costs. Since peer mentors are usually individuals who have successfully coped with the specified disease, they can be instrumental in identifying life goals and problem-solving $(39,49)$, as well as reducing pressure on providers (50) to assess and resolve ancillary life issues related to healthcare utilization costs.

As an exploratory pilot study, PALS is not exempt of limitations, which we have carefully considered and used as learning experiences to further refine the intervention program going forward. First, there was not a control group, which limits our ability to determine the effectiveness of the peer mentoring program compared with usual care of lupus patients. However, cost estimates two months before and two months after the intervention allowed evaluation of incurred costs with and without the intervention. Also, costs associated with other medical facilities and paid caregivers were not examined, along with direct nonmedical care costs such as patient transportation, childcare if needed during visits, and costs incurred by other family members who may have been involved in transporting patients to visits. We were also unable to obtain indirect costs including costs associated with lost wages incurred from taking time off work for intervention visits. We also did not assess current medications, which could impact findings related to disease activity. Lastly, we did not collect patient reported outcomes from our mentors, and so were unable to assess how the program impacted their health and associated costs. All of these limitations have been addressed and corresponding information will be collected in the expanded PALS randomized clinical trial. Additional limitations that could potentially contribute to undetected bias include the study design, selection of a distinct SLE population who may be of higher education and income than the general SLE population, small sample size, and lack of multivariate analysis.

Overall, we were able to show that a peer mentoring program for AA women with lupus incorporating structured education and support improves relevant outcomes and reduces hospitalization costs. This suggests that if adopted on a larger scale, this approach has the potential to significantly reduce financial burden on both patients and medical facilities, as well as improve the quality of life of a high-risk patient population. 


\section{Funding}

This work was supported by the South Carolina Clinical \& Translational Research (SCTR) Institute, with an academic home at the Medical University of South Carolina CTSA, NIH/NCATS Grant Number UL1 TR001450 and the Rheumatology and Immunology MCRC NIH/NIAMS Grant Number AR062755.

\section{References}

1. Kan H, Song X, Johnson B, Bechtel B, O'Sullivan D, Molta C. Healthcare utilization and costs of systemic lupus erythematosus in Medicaid. Biomed Res Int. 2013;2013.

2. Narayanan S, Wilson K, Ogelsby A, Juneau P, Durden E. Economic burden of systemic lupus erythematosus flares and comorbidities in a commercially insured population in the United States. J Occup Environ Med. 2013;55(11):1262-70. [PubMed: 24202242]

3. Zhu T, Tam L, Li E. Cost-of-illness studies in systemic lupus erythematosus: A systematic review. Arthritis Care Res (Hoboken). 2011;63(5):751-60. [PubMed: 21557530]

4. Brown EA, Brinton D, Gadsden BK, Simpson KN. Burden of Illness and Avoidable Costs for People Hospitalized with Systemic Lupus Erythematosus: Impact of Inadequate Access to Primary Care. AcademyHealth; New Orleans, LA2017.

5. Slawsky KA, Fernandes AW, Fusfeld L, Manzi S, Goss TF. A structured literature review of the direct costs of adult systemic lupus erythematosus in the US. Arthritis Care Res (Hoboken). 2011;63(9):1224-32. [PubMed: 21584945]

6. Pons-Estel G, Alarcón G, Scofield L, Reinlib L, Cooper G. Understanding the Epidemiology and Progression of Systemic Lupus Erythematosus. Seminars in Arthritis and Rheumatism. 2010;39(4): 257-68. [PubMed: 19136143]

7. Feldman C, Bermas B, Zibit M, Fraser P, Todd D, Fortin P, et al. Designing an intervention for women with systemic lupus erythematosus from medically underserved areas to improve care: a qualitative study. Lupus. 2013;22(1):52-62. [PubMed: 23087258]

8. Anderson A, Damio G, Chapman D, Perez-Escamilla R. Differential response to an exclusive breastfeeding peer counseling intervention: the role of ethnicity Journal of Human Lactation 2007;23(1):16-23.

9. Malchodi C, Oncken C, Dornelas E, Caramanica L, Gregonis E, Curry S. The effects of peer counseling on smoking cessation and reduction. Obstretics and Gynecology. 2003;101(3):504-10.

10. Woodbury M, Botros M, Kuhnke J, Greene J. Evaluation of a peer-led self-management education programme PEP talk: Diabetes, healthy feet and you. Int Wound J. 2013;10(6):703-11. [PubMed: 26074389]

11. Philis-Tsimikas A, Fortmann A, Lleva-Ocana L, Walker C, Gallo L. Peerled diabetes education programs in high-risk Mexican Americans improve glycemic control compared with standard approaches: a Project Dulce promotora randomized trial. Diabetes care. 2011;34:1926-31 [PubMed: 21775748]

12. Goldenberg D, Payne L, Hayes L, Zeltzer L, Tsao J. Peer mentorship teaches social tools for pain self-management: A case study. J Pain Manag. 2013;6(1):61-8. [PubMed: 25383136]

13. Sandhu S, Veinot P, Embuldeniya G, Brooks S, Sale J, Huang S, et al. Peer-to-peer mentoring for individuals with early inflammatory arthritis: feasibility pilot. BMJ Open. 2013;3(3).

14. Lorig K, Chastain R, Ung E, Shoor S, Holman H. Development and evaluation of a scale to measure perceived self-efficacy in people with arthritis. Arthritis \& Rheumatism. 1989;32(1):3744. [PubMed: 2912463]

15. Williams EM, Hyer JM, Viswanathan R, Faith TD, Voronca D, Gebregzaibher M, et al. Peer-toPeer Mentoring for African American Women With Lupus: A Feasibility Pilot. Arthritis Care Res (Hoboken). 2018;70(6):908-17. [PubMed: 29161471]

16. Lorig K, Sobel D, Stewart A. Evidence that a chronic disease self-management program can improve health status while reducing utilization and costs: A randomized trial. Medical Care. 1999;37(1):5-14. [PubMed: 10413387]

17. Lorig K, Holman H. Arthritis Self-Management Studies: A twelve-year review. Health Education Quarterly 1993;20(1):17-28. [PubMed: 8444622] 
18. Foundation Arthritis. The Systemic Lupus Erythematosus Self-Help Course: Program guidelines and procedures manual. Atlanta, GA1987.

19. Williams E, Bruner L, Penfield M, Kamen D, Oates J. Stress and Depression in Relation to Functional Health Behaviors in African American Patients with Systemic Lupus Erythematosus. Rheumatology: Current Reports. 2014(Supp 4).

20. Williams E, Penfield M, Kamen D, Oates J. An Intervention to Reduce Psychosocial and Biological Indicators of Stress in African American Lupus Patients: The Balancing Lupus Experiences with Stress Strategies Study. Open Journal Of Preventive Medicine. 2014;4(1):22-31. [PubMed: 24999444]

21. Williams E, Kamen D, Penfield M, Oates J. Stress Intervention and Disease in African American Lupus Patients: The Balancing Lupus Experiences with Stress Strategies (BLESS) Study. Health. 2014;6(1):71-9. [PubMed: 24932382]

22. McElhone K, Abott J, Shelmerdine J, Bruce I, Ahmad Y, Gordon C, et al. Development and validation of a disease-specific health-related quality of life measure, the LupusQol, for adults with systemic lupus erythematosus. Arthritis \& Rheumatism. 2007;57(6):972-9. [PubMed: 17665467]

23. Hibbard J, Stockard J, Mahoney E, Tusler M. Development of the Patient Activation Measure (PAM): conceptualizing and measuring activation in patients and consumers. Health Serv Res 2004;39(4 Pt 1):1005-26. [PubMed: 15230939]

24. Bae S, Koh H-K, Chang D-K, Kim M-H, Part J-K, Kim S-Y. Reliability and validity of systemic lupus activity measure-revised (SLAM-R) for measuring clinical disease activity in systemic lupus erythematosus. Lupus. 2002;10:405-9.

25. Williams EM, Hyer JM, Viswanathan R, Faith TD, Egede L, Oates JC, et al. Cytokine balance and behavioral intervention; findings from the Peer Approaches to Lupus Self- Management (PALS) project. Hum Immunol. 2017;78(9):574-81 [PubMed: 28716698]

26. Kroenke K, Spitzer R. The PHQ-9: A new depression and diagnostic severity measure. Psychiatric Annals. 2002;32(9):509-21.

27. Spitzer R, Kroenke K, Williams J, Loewe B. A brief measure for assessing generalized anxiety disorder: the GAD-7. Archives of Internal Medicine. 2006;166(10):1092-7. [PubMed: 16717171]

28. Whitley E, Valverde P, Wells K, Williams L, Teschner T, Shih YC. Establishing common cost measures to evaluate the economic value of patient navigation programs. Cancer. 2011;117(15 Suppl):3618-25. [PubMed: 21780096]

29. Sanders GD, Neumann PJ, Basu A, Brock DW, Feeny D, Krahn M, et al. Recommendations for Conduct, Methodological Practices, and Reporting of Cost-effectiveness Analyses: Second Panel on Cost-Effectiveness in Health and Medicine. JAMA. 2016;316(10):1093-103. [PubMed: 27623463]

30. Polinder STH, Panneman M, van Beeck E. Methodological appraoches for cost-effectiveness and cost-utility analysis of injury prevention measures. World Health Organization; 2011.

31. Rotheram-Borus M, Richter L, van Heerden A, van Rooyen H, Tomlinson M, Harwood J, et al. A cluster randomized controlled trial evaluating the efficacy of peer mentors to support South African women living with HIV and their infants. PLoS One. 2014;9(1):e84867. [PubMed: 24465444]

32. Jerson B, D’Urso C, Arnon R, Miloh T, Iyer K, Kerkar N, et al. Adolescent transplant recipients as peer mentors: a program to improve self-management and health-related quality of life. Pediatr Transplant. 2013;17(7):612-20. [PubMed: 23905874]

33. Thomas R, Lorenzetti D, Spragins W. Systematic review of mentoring to prevent or reduce tobacco use by adolescents. Acad Pediatr. 2013;13(4):300-7. [PubMed: 23830017]

34. Dorgo S, Robinson K, Bader J. The effectiveness of a peer-mentored older adult fitness program on perceived physical, mental, and social function. J Am Acad Nurse Pract. 2009;21(2):116-22. [PubMed: 19228250]

35. Eskicioglu P, Halas J, Senechal M, Wood L, McKay E, Villeneuve S, et al. Peer mentoring for type 2 diabetes prevention in first nations children. Pediatrics. 2014;133(6):e1624-e31. [PubMed: 24819579]

36. Heisler M, Piette J. "I help you, and you help me": facilitated telephone peer support among patients with diabetes. The Diabetes Educator. 2005;31:869-79. [PubMed: 16288094] 
37. Knox L, Huff J, Graham D, Henry M, Bracho A, Henderson C, et al. What peer mentoring adds to already good patient care: Implementing the Carpeta Roja peer mentoring program in a wellresourced health care system. Ann Fam Med. 2015;13(Suppl 1):S59-565. [PubMed: 26304973]

38. Long J, Jahnle E, Richardson D, Loewenstein G, Volpp K. Peer mentoring and financial incentives to improve glucose control in African American veterans: a randomized trial. Ann Intern Med. 2012;156(6):416-24. [PubMed: 22431674]

39. Williams EM, Egede L, Faith T, Oates J. Effective Self-Management Interventions for Patients With Lupus: Potential Impact of Peer Mentoring. Am J Med Sci. 2017;353(6):580-92. [PubMed: 28641721]

40. Khamashta MA, Bruce IN, Gordon C, Isenberg DA, Ateka-Barrutia O, Gayed M, et al. The cost of care of systemic lupus erythematosus (SLE) in the UK: annual direct costs for adult SLE patients with active autoantibody-positive disease. Lupus. 2014;23(3):273-83. [PubMed: 24356612]

41. Oglesby A, Korves C, Laliberte F, Dennis G, Rao S, Suthoff ED, et al. Impact of early versus late systemic lupus erythematosus diagnosis on clinical and economic outcomes. Appl Health Econ Health Policy. 2014;12(2):179-90. [PubMed: 24573911]

42. Billings J, Anderson GM, Newman LS. Recent findings on preventable hospitalizations. Health Aff (Millwood). 1996;15(3):239-49.

43. Gaskin DJ, Hoffman C. Racial and ethnic differences in preventable hospitalizations across 10 states. Medical Care Research and Review. 2000;57(1_suppl):85-107. [PubMed: 11092159]

44. Ward MM. Avoidable hospitalizations in patients with systemic lupus erythematosus. Arthritis Rheum. 2008;59(2):162-8. [PubMed: 18240192]

45. Quality AfHRa. Strategy 2: Communicating to Improve Quality 2017 [Available from: https:// www.ahrq.gov/professionals/systems/hospital/engagingfamilies/strategy2/index.html.

46. Portnoy JM, Waller M, De Lurgio S, Dinakar C. Telemedicine is as effective as in-person visits for patients with asthma. Ann Allergy Asthma Immunol. 2016;117(3):241-5. [PubMed: 27613456]

47. Isetta V, Negrin MA, Monasterio C, Masa JF, Feu N, Alvarez A, et al. A Bayesian costeffectiveness analysis of a telemedicine-based strategy for the management of sleep apnoea: a multicentre randomised controlled trial. Thorax. 2015;70(11):1054-61. [PubMed: 26310452]

48. Hirshkowitz M, Sharafkhaneh A. A telemedicine program for diagnosis and management of sleepdisordered breathing: the fast-track for sleep apnea tele-sleep program. Semin Respir Crit Care Med. 2014;35(5):560-70. [PubMed: 25353101]

49. Heisler M Different models to mobilize peer support to improve diabetes self-management and clinical outcomes: evidence, logistics, evaluation considerations and needs for future research. Fam Pract. 2010;27 Suppl 1:i23-32. [PubMed: 19293400]

50. Law G, Pope J, Lalani S, Silverman E, Cooper G, Fortin P, Zummer M, Smith C, Petty R, Tucker L, Albert L, Huber A, Ramsey S, Arbillaga H, Chedeville G, Hudson M, CaNIOS Investigators, Peschken C. Barriers To Healthcare in a Multiethnic Cohort of Systemic Lupus Erythematosus (SLE) Patients: Patient and Physician Perceptions. Romanian Journal of Rheumatology. 2010;19(1):12-9. 
Table 1:

\section{Weekly Session Content}

\begin{tabular}{|c|c|c|}
\hline Week & Session Topic & Detailed Content \\
\hline 1 & Goal setting/ action planning & Introduction to SLE management and a chance for the mentee to get to know the mentor \\
\hline 2 & Exercise & Appropriate exercise for maintaining and improving strength, flexibility, and endurance \\
\hline 3 & Medication overview & Appropriate use of medications and evaluation of new treatments/ making informed decisions \\
\hline 4 & Effective communication & Communicating effectively with family, friend, and health professionals \\
\hline 5 & Nutrition/ healthy eating & Sessions five will stress nutrition and healthy eating \\
\hline 6 & Stress relaxation techniques & $\begin{array}{l}\text { Relaxation techniques to cope with chronic pain, manage sudden increases in pain and other } \\
\text { symptoms and reduce flares }\end{array}$ \\
\hline 7 & Coping & $\begin{array}{l}\text { Coping, including problem solving and strategies for coping with pain, other lupus symptoms, and } \\
\text { interpersonal issues }\end{array}$ \\
\hline 8 & Body image & $\begin{array}{l}\text { Body image issues, including concerns around hair loss, skin discoloration and scaring, and } \\
\text { medication-induced weight gain }\end{array}$ \\
\hline 9 & Complications & $\begin{array}{l}\text { Complications (e.g. kidney disease, digestive issues, pregnancy outcomes) fatalism-fear of } \\
\text { complications and how that impacts perception of survival, and self-efficacy }\end{array}$ \\
\hline 10 & Self-monitoring & Self-monitoring and depression \\
\hline 11 & Sexuality/ sexual health & Sexuality/ sexual health, including spousal interactions and dating \\
\hline 12 & Trust & $\begin{array}{l}\text { Perceptions of care from doctor(s), impact on adherence, seeking second opinions, perceived } \\
\text { discrimination, and perceived cultural competence of provider(s); Review of material from all previous } \\
\text { sections; Feedback on program, discussion of any progress made }\end{array}$ \\
\hline
\end{tabular}

Lupus. Author manuscript; available in PMC 2020 July 01. 
Table 2:

Baseline characteristics of Mentees and Mentors

\begin{tabular}{llll}
\hline Variable & Mentee N (\%) & Mentor N (\%) & P value \\
\hline Age (years) & & & 0.39 \\
$<25$ & $2(8.7)$ & $0(0.0)$ & \\
$25-34$ & $2(8.7)$ & $3(30.0)$ & \\
$35-44$ & $8(34.8)$ & $3(30.0)$ & \\
$45-54$ & $5(21.7)$ & $1(10.0)$ & \\
$55-64$ & $1(4.4)$ & $2(20.0)$ & \\
$>65$ & $5(21.7)$ & $1(10.0)$ & \\
\hline Marital Status & & & 0.15 \\
Married & $2(8.7)$ & $3(30.0)$ & \\
Other & $21(91.3)$ & $8(80.0)$ & \\
\hline Education & & & \\
$<$ High School & $3(13.7)$ & $0(0.0)$ & \\
High School & $2(9.1)$ & $8(80.0)$ & \\
Some College & $3(13.7)$ & $0(0.0)$ & \\
College Graduate & $14(63.6)$ & $8(80.0)$ & \\
\hline Income & & & \\
$<\$ 15,000$ & $5(21.7)$ & $0(0.0)$ & \\
\$15,000-34,999 & $6(26.1)$ & $3(33.3)$ & \\
\$35,000-64,999 & $5(21.7)$ & $1(11.1)$ & \\
\$65,000 & $2(8.7)$ & $4(44.4)$ & \\
Other/No response & $5(21.7)$ & $1(11.1)$ & \\
\hline Renal involvement & & & \\
History of transplant & $3(13.7)$ & & \\
History of dialysis & $2(9.1)$ & & \\
Lupus Nephritis & $5(21.7)$ & $5(50.0)$ & \\
\hline Flares in last 3 months & & & \\
None & $6(26.1)$ & & \\
Mild & $6(26.1)$ & & \\
Moderate & $7(30.4)$ & & \\
Severe & $4(17.4)$ & & \\
\hline & & & \\
\hline
\end{tabular}

Lupus. Author manuscript; available in PMC 2020 July 01. 
Table 3:

Costs of Implementing the Peer Mentoring Program

\begin{tabular}{lll}
\hline Variable & Cost Per Mentee & Cost Per Week Per Mentee \\
\hline Lab Fees & $\$ 35.75$ & $\$ 2.97$ \\
\hline Research Support & $\$ 40.00$ & $\$ 3.33$ \\
\hline Consultant/Contractual & $\$ 60.00$ & $\$ 5.00$ \\
\hline Participant Incentives & $\$ 572.50$ & $\$ 47.71$ \\
\hline Cell Phones & $\$ 494.58$ & $\$ 24.73$ \\
\hline Supplies & $\$ 88.67$ & $\$ 7.39$ \\
\hline Total & $\$ 1882.83$ & $\$ 91.14$ \\
\hline
\end{tabular}


Table 4:

Outcomes of the Peer Mentoring Program

\begin{tabular}{lllll}
\hline Outcome & Pre-Intervention Mean (95\% CI) & Post-Intervention Mean (95\% CI) & P value & C/E \\
\hline Depression & $7.7(5.25,10.14)$ & $5.4(3.57,7.22)$ & 0.024 & $\$ 818$ \\
\hline Anxiety & $1.75(1.11,2.39)$ & $1.2(0.75,1.64)$ & 0.017 & $\$ 3,423$ \\
\hline Disease Activity (SLAQ) & $28.2(13.83,42.57)$ & $6.25(5.20,7.30)$ & 0.004 & $\$ 85.74$ \\
\hline & & & BCR \\
\hline Healthcare Costs & $\$ 24,289(55.51,48524)$ & $\$ 872(-324,2070)$ & 0.047 & 12.44 \\
\hline
\end{tabular}

